\title{
Fahrkompetenz und Morbus Parkinson
}

\author{
Ein Update
}

\section{Driving ability and Parkinson's disease}

An update

\author{
Vanessa Andelfinger ${ }^{1}$, Matthias Weisbrod ${ }^{2,3}$, \\ Michael Fritz ${ }^{4}$, Steffen Aschenbrenner ${ }^{1}$ \\ 1 Abteilung für Klinische Psychologie und Neuropsycholo- \\ gie SRH Klinikum Karlsbad \\ 2 Abteilung für Psychiatrie und Psychotherapie SRH Klini- \\ kum Karlsbad \\ 3 Klinik für allgemeine Psychiatrie, Zentrum für Psychosozi- \\ ale Medizin, Universitätsklinikum Heidelberg \\ 4 Abteilung für Neurologie SRH Klinikum Karlsbad
}

\begin{abstract}
ZUSAMMENFASSUNG
Diese Arbeit gibt einen Überblick über die wissenschaftliche Studienlage zum Thema Fahrkompetenz bei Morbus Parkinson. Neben der Darstellung der geltenden Rechtslage erfolgt eine Übersicht über motorische, kognitive, visuelle und dopaminassoziierte Symptome, die Zweifel an der Fahrkompetenz von Parkinson-Patienten begründen können. In diesem Kontext findet eine differenzierte Betrachtung von Fahrverhaltensproben und Fahrsimulatoren statt. Den Abschluss bilden klinische Handlungsempfehlungen für Patienten und Behandler, die Betrachtung medikamentöser Behandlung, operative Behandlungsansätze und neuropsychologische Therapiemöglichkeiten.
\end{abstract}

\section{Einleitung}

Die Fahrkompetenz ist ein wesentlicher Bereich des täglichen Lebens, der durch die körperlichen, visuellen und kognitiven Beeinträchtigungen bei Patienten mit Morbus Parkinson eingeschränkt sein kann [1, 2]. Die eigenständige Nutzung eines Fahrzeugs berührt die Erreichbarkeit medizinischer Versorgung, bis hin zum Erhalt der Unabhängigkeit sowie dem Identitäts- und Kompetenzgefühl von Erkrankten [3, 4]. Parkinson-Patienten sind im Vergleich zu altersgleichen Fahrern stärker in ihrer Fahrkompetenz beeinträchtigt, wobei sich besonders ein höheres Alter und der Abbau kognitiver Fähigkeiten negativ auf die Fahrkompetenz auswirken [5, 6]. Eine etablierte Möglichkeit das Kompensationspotenzial der Patienten abzuschätzen, ist die Fahrverhaltensprobe [7]. Auch neuere Generationen von Fahrsimulatoren können in diesem Bereich unterstützend wirken.

\section{Rechtliche Aspekte}

Die Fahrerlaubnis ist von der körperlichen und geistigen Eignung abhängig ( $§ 2$ Abs 4 StVG). Dabei hat jeder Verkehrsteilnehmer die Pflicht, seine Eignung zur Teilnahme am Straßenverkehr selbstständig zu prüfen und für die Nichtgefährdung eigenständig Vorsorge zu tragen ( $§ 2$ Abs $1 \mathrm{FeV}$ ). Wie in Anlage 4 der Fahrerlaubnis-Verordnung (FeV) geregelt, betrifft dies Parkinson-Patienten besonders. Die Eignung zum Führen von Fahrzeugen der Gruppe 2 (LKW, Personenbeförderung) ist bei M. Parkinson prinzipiell nicht gegeben. Bei Fahrzeugen der Gruppe 1 besteht bei leichten Fällen und einer erfolgreichen The- rapie die (bedingte) Eignung. Nachuntersuchungen sind bei bedingter Eignung in Abständen von 1, 2 und 4 Jahren erforderlich. Eine extrapyramidale Erkrankung kann nach den Begutachtungsleitlinien zur Kraftfahreignung (BASt) eine Herabsetzung der Leistungs- und Belastungsfähigkeit zur Folge haben. Bestehen Zweifel an der Fahreignung wird eine neurologische Begutachtung und, je nach Umständen, eine psychologische Zusatzuntersuchung und ggf. eine praktische Fahrverhaltensbeobachtung empfohlen, mit Nachuntersuchungen im Einzelfallbefund. Die Führerscheinbehörde muss ihr bekannt gewordenen Zweifeln stets nachgehen und kann ggf. eine medizinisch-psychologische Untersuchung bei einer zertifizierten Einrichtung anordnen. Sowohl Patient als auch Behandler stehen für die Einhaltung dieser Verordnung in der Pflicht (BGH, NJW 2003, 2309). Dem Behandler obliegen eine ausreichende Aufklärung, Information und ggf. Überwachung des Patienten. Kommt der Behandler dem nicht, nur unzureichend oder oberflächlich nach, kann es zu Schadenersatz- und Schmerzensgeldansprüchen kommen ( $\S 611,280,253$ $B G B)$. Zur haftungsrechtlichen Absicherung ist eine umfassende Dokumentation entscheidend [8]. Dem Patienten drohen bei Hinwegsetzen über die Empfehlungen neben Einbußen im Versicherungsschutz bei einer konkreten Gefährdung laut Strafgesetzbuch auch Geld- oder Freiheitsstrafen (§316 StGB). 


\section{Motorische, kognitive und \\ visuelle Parkinsonsymptome und Fahrkompetenz}

Die Einschränkung der Fahrkompetenz steht bei Parkinson-Patienten in Verbindung mit motorischen, exekutiven, visuellen und visuell-räumlichen Funktionseinbußen [9]. Motorische Hauptsymptome, die zu einer relevanten Einschränkung der Fahrkompetenz führen, sind Akinese, Tremor und Dyskinesien sowie ausgeprägte On-/Off-Phänomene [2]. Bezogen auf den Schweregrad der motorischen Erkrankung ist die Stufe 3 der Hoehn \& Yahr Skala ein Prädiktor für eine mangelnde Fahrkompetenz [10].

Nicht motorische Symptome der Parkinsonkrankheit können bereits Jahre vor den motorischen Symptomen einsetzen $[11,12]$ und mit steigender Krankheitsdauer zunehmen [13, 14]. Schlechtere Leistungen in Aufmerksamkeit, der Aufnahme und Verarbeitung visueller sowie visuell-räumlicher Informationen und in Teilbereichen exekutiver Funktionen sind bei Parkinson-Patienten mit mehr Fahrfehlern und Veränderungen im Fahrverhalten assoziiert [5, 9, 15-17]. Zusätzliche Aufgaben, z. B. visuelles Suchen, können zu einer kognitiven Überlastung [16, 18] und zu einem erhöhten Unfallrisiko führen [19]. Betrachtet man die Demenzerkrankung, ist besonders bei mittleren-schweren Ausprägungen die Fahrkompetenz beeinträchtigt [20].

Anforderungen an die visuelle Leistungsfähigkeit werden in Anlage 6 der FeV geregelt. Zu den bei Parkinson-Patienten auftretenden Funktionsstörungen zählen die Verschlechterung der Kontrastsensitivität und Veränderungen in der Farbwahrnehmung. Hinzu kommen Schwierigkeiten in der visuellen Wahrnehmung [21]. Insbesondere geringere Kontrastsensitivität ist mit einer verminderten Fahrleistung assoziiert. Sie führt zu einer schlechteren Fahrzeugkontrolle, mehr Sicherheitsfehlern und langsameren Reaktionszeiten [15, 17].

Eine präzise Beurteilung der Fahrkompetenz gestaltet sich im Einzelfall schwierig. Keines der eingesetzten Untersuchungsverfahren erreicht das höchste Level der „eindeutigen Vorhersagbarkeit“ [22]. In Deutschland gibt es kein etabliertes standardisiertes Vorgehen zur Bestimmung der Fahrkompetenz bei Parkinson-Patienten. Für eine objektive Bewertung wäre eine standardisierte Beurteilung jedoch wünschenswert [23]. Entscheidungen über die Fahrkompetenz bleiben somit Einzelfallentscheidungen. Ansätze, Cut-off-Werte auf der Basis von kognitiven Beeinträchtigungen (z. B. Aufmerksamkeitsleistungen und demenziellen Erkrankungen), motorischen und visuell-sensorischen Funktionen sowie Indizes zum Schweregrad der Parkinsonerkrankung (z. B. Krankheitsdauer) festzulegen $[24,25]$, sind vom Einsatz in der klinischen Routine noch weit entfernt. Einen Überblick über die aktuelle Studienlage liefert $>$ Tab. 1.

\section{Fahrverhaltensbeobach- tung im Straßenverkehr und Fahrsimulatorstudien}

Fahrverhaltensbeobachtungen (FVB) stellen ökologisch valide Maße der Fahrkompetenz dar. Hier erhält der Fahrer externe Hinweisreize zur Steuerung des Fahrzeugs [12, 19]. In Simulatoren können identische Anforderungen auf einer standardisierten Route festgelegt oder ein direktes Unfallrisiko simuliert werden [6], sie erfassen das Fahrverhalten präzise in einer kontrollierten und reproduzierbaren Umgebung $[12,19]$. Bei FVBs gilt es zudem variierende Fahrzeugausstattungen $[17,26,27]$ und die unterschiedlichen zeitlichen Intervalle, in denen die FVBs stattfinden [10, 27, 28], zu beachten. Weiter besteht eine Heterogenität in den verwendeten Methoden zur Bewertung der Fahrkompetenz und -leistung [5, 17, 26]. Ein Nachteil von Simulatoren ist die große Bandbreite zwischen deren Eigenschaften und der Validität gegenüber dem reellen Straßenverkehr [19]. So ist die Verwendung des Begriffs Simulator nicht einheitlich definiert und kann verschiedene Systeme umfassen [15, 16, 29]. Auch wenn die Quote des Nichtbestehens bei FVBs bei Parkinson-Patienten höher ist als im Simulator, sind beide Methoden zur Erfassung der Fahrsicherheit geeignet [6]. Dabei sind Simulatoren besonders bei älteren Fahrern nützliche Werkzeuge [30]. Annahme ist: Je ähnlicher die Simulation dem reellen Straßenverkehr, desto geringer die Unterschiede.

\section{Klinische Handlungsempfehlungen}

\section{Patienten und Behandler}

Bei bestehenden Zweifeln an der Fahrkompetenz ist eine kooperative Zusammenarbeit von Patienten und Behandler entscheidend. Auf dem Boden einer umfassenden Aufklärung obliegt es dem Arzt, die Eigenverantwortung des Patienten deutlich zu machen. Ein verbessertes Problembewusstsein dient so dem Schutz des Patienten sowie der Allgemeinheit [23]. Dabei unterliegt der Behandler weiterhin der Schweigepflicht, welche nur durch einen „gerechtfertigten Notstand“ verletzt werden darf [8]. Einen Überblick über Symptome, die Zweifel an der Fahrkompetenz begründen können, führt $\triangleright$ Tab. 2 auf. Bestehen Zweifel an der Fahrkompetenz kann eine informelle Abklärung erfolgen. Neben der Beurteilung durch einen neurologischen Facharzt sind für eine differenzierte und patientengerechte Behandlung bei visuellen Problemen die Beurteilung durch einen Ophthalmologen und bei kognitiven Problemen eine Testung mit geeigneten psychologischen Testverfahren zu empfehlen. Bei motorischer Eignung sollte unbedingt die kognitive Leistungsfähigkeit erfasst werden. Bei einer Abklärung der Fahrkompetenz über eine standardisierte FVB kann der Behandler seine Entscheidung über die Fahrkompetenz auf diese stützen. 
\ Tab. 1 Überblick der Studienlage zur Vorhersage der Fahrkompetenz bei Parkinson-Patienten.

\begin{tabular}{|c|c|c|c|}
\hline Autoren & Stichprobe & Methode & Hauptaussage \\
\hline $\begin{array}{l}\text { Classen, Brumback, Cra- } \\
\text { wford et al. } 2019 \text { [23] }\end{array}$ & $\begin{array}{l}\mathrm{PD} n=101 \\
\mathrm{KG} n=138\end{array}$ & FVB, UFOV RI & Cut-point von 3 im UFOV RI: Prädiktor für Leistung in FVB. \\
\hline $\begin{array}{l}\text { Uc, Rizzo, O‘Shea et al. } \\
2017 \text { [14] }\end{array}$ & $\begin{array}{l}\mathrm{PD} n=67 \\
\mathrm{KG} n=110\end{array}$ & FVB, kognitive Tests & Kognitive Einbußen und Anstieg Fehlerraten an t2: PD>KG. \\
\hline $\begin{array}{l}\text { Uc, Rizzo, Johnson et al. } \\
2009 \text { [5] }\end{array}$ & $\begin{array}{l}\text { PD } n=84 \text { (median } \mathrm{H} \& \mathrm{Y} 2 \text { ) } \\
\mathrm{KG} n=182\end{array}$ & FVB, kognitive Tests & $\begin{array}{l}\text { Sicherheitsfehler: PD > KG. Variabilität der Fahrsicherheit groß bei PD. } \\
\text { Beeinträchtigungen der visuellen Wahrnehmung und Kognition mit } \\
\text { Fahrsicherheitsfehlern assoziiert. }\end{array}$ \\
\hline $\begin{array}{l}\text { Cordell, Lee, Granger et } \\
\text { al. } 2008 \text { [26] }\end{array}$ & $\begin{array}{l}P D n=53(H \& Y 1-3) \\
K G n=129\end{array}$ & $\begin{array}{l}\text { FVB, klinische } \\
\text { Einschätzung }\end{array}$ & Fehler bei FVB: PD > KG. \\
\hline $\begin{array}{l}\text { UC, Rizzo, Anderson et } \\
\text { al. } 2007 \text { [27] }\end{array}$ & $\begin{array}{l}\mathrm{PD} n=77(H \& Y 1-3) \\
K G n=152\end{array}$ & FVB, kognitive Tests & Navigations- und Sicherheitsfehler: PD> KG. \\
\hline $\begin{array}{l}\text { Ranchet, Morgan, Akin- } \\
\text { wuntan et al. } 2020 \text { [18] }\end{array}$ & $\begin{array}{l}P D n=19(H \& Y 2-3) \\
K G n=14\end{array}$ & $\begin{array}{l}\text { Simulator, } \\
\text { kognitive Tests }\end{array}$ & Detektion stationäre Reize: PD<KG. \\
\hline $\begin{array}{l}\text { Ranchet, Broussolle, } \\
\text { Paire-Ficout } 2016 \text { [16] }\end{array}$ & $P D n=16(H \& Y 1-3)$ & $\begin{array}{l}\text { Simulator, } \\
\text { kognitive Tests }\end{array}$ & $\begin{array}{l}\text { PD-Patienten erleben über die Zeit einen Rückgang der kognitiven } \\
\text { Flexibilität, welcher mit Veränderungen im Fahrverhalten assoziiert ist. }\end{array}$ \\
\hline $\begin{array}{l}\text { Devos, Vandenberg, } \\
\text { Nieuwboer } 2013 \text { [24] }\end{array}$ & $P D n=60$ & $\begin{array}{l}\text { FVB, } \\
\text { Screening-Batterie }\end{array}$ & $\begin{array}{l}\text { Screening-Batterie identifiziert alle Fahrer, bei denen Fahrkompetenz } \\
\text { besteht. Ein Teil der PD, die die Batterie nicht bestehen, besteht FVB. }\end{array}$ \\
\hline $\begin{array}{l}\text { Devos, Vandenberg, Tant } \\
\text { et al. } 2013 \text { [9] }\end{array}$ & $P D n=104(H \& Y 2-3)$ & FVB, kognitive Tests & $\begin{array}{l}\text { Schlechtere Leistung in der FVB sind mit schlechteren Leistungen in } \\
\text { Motorik, visuellen/visuell-räumlichen Fähigkeiten, Aufmerksamkeitsleis- } \\
\text { tungen und exekutiven Funktionen assoziiert. }\end{array}$ \\
\hline $\begin{array}{l}\text { Thiri Kyaw, Nishikawa, } \\
\text { Moritoyo et al. } 2013 \text { [28] }\end{array}$ & $P D n=42(H \& Y 2-3)$ & Simulator & $\begin{array}{l}\text { UPDRS allein reicht nicht aus, um Fahrkompetenz bei } \\
\text { PD vorherzusagen. }\end{array}$ \\
\hline $\begin{array}{l}\text { Uc, Rizzo, Anderson et al. } \\
2009[15]\end{array}$ & $\begin{array}{l}P D n=67(H \& Y 1-3) \\
K G n=51\end{array}$ & $\begin{array}{l}\text { Simulator, } \\
\text { kognitive Tests }\end{array}$ & $\begin{array}{l}\text { Unter schlechten Sichtverhältnissen zeigen PD schlechtere Fahrzeug- } \\
\text { kontrolle und erhöhtes Unfallrisiko. }\end{array}$ \\
\hline $\begin{array}{l}\text { Amick, Grace, Ott } 2007 \\
\text { [17] }\end{array}$ & $P D n=25$ & FVB, kognitive Tests & $\begin{array}{l}\text { Multifaktorielle neuropsychologische Tests, die visuelle/visuell } \\
\text { räumliche Fähigkeiten erfordern, sind bestes Screening für riskantes } \\
\text { Fahrverhalten bei PD. }\end{array}$ \\
\hline $\begin{array}{l}\text { Singh, Pentland, Hunter } \\
\text { et al. } 2007 \text { [10] }\end{array}$ & $P D n=154(H \& Y 1-3)$ & FVB, RZ-Messung & $\begin{array}{l}\text { Faktoren zur Unterscheidung von Fahrsicherheit: H\&Y 3, höheres Alter, } \\
\text { Komorbiditäten, RZ, schlechtere Leistungen in der FVB. }\end{array}$ \\
\hline
\end{tabular}

$\mathrm{ADL}=$ activities of daily living, $\mathrm{H} \& \mathrm{Y}=$ Hoehn und Yahr Skala, $\mathrm{FVB}=$ Fahrverhaltensbeobachtung, $\mathrm{KG}=$ Kontrollgruppe, $\mathrm{LVC}=$ lane violation counts, $\mathrm{PD}=$ Parkinson Disease Gruppe, RZ= Reaktionszeit, SDLP = standard deviation of lateral position, UPDRS= Unified Parkinson's Disease Rating Scale, $\mathrm{t} 2$ = Testzeitpunkt 2, UVOF RI = useful field of view risk index.

- Tab. 2 Motorische, kognitive, visuelle und dopaminassoziierte Symptome, die Zweifel an der Fahrkompetenz von Parkinson-Patienten begründen können.

\begin{tabular}{|c|c|c|c|}
\hline Motorisch & Kognitiv & Visuell & Dopaminassoziierte Symptome \\
\hline $\begin{array}{l}\text { - Motorische } \\
\text { Verlangsamung } \\
\text { - Akinese/Freezing } \\
\text { - Tremor } \\
\text { - Dyskinesien } \\
\text { - On-/Off-Phänomene }\end{array}$ & $\begin{array}{l}\text { - Kognitive Verlangsamung } \\
\text { - Verlangsamung der Reaktionszeit } \\
\text { - Verminderung der Belastbarkeit } \\
\text { - Störungen der Daueraufmerksamkeit } \\
\text { - Störungen der selektiven Aufmerksamkeit } \\
\text { - Störungen der geteilten Aufmerksamkeit } \\
\text { - (Arbeits-) Gedächtnisstörungen } \\
\text { - Störungen der kognitiven Flexibilität } \\
\text { - Inhibitionsstörungen } \\
\text { - Störungen der Verarbeitung räumlicher Informationen } \\
\text { - Mittlere bis schwere Formen demenzieller Erkrankungen }\end{array}$ & $\begin{array}{l}\text { - Verminderung der } \\
\text { Kontrastsensitivität } \\
\text { - Deutliche } \\
\text { Visusminderung } \\
\text { - Störungen in der } \\
\text { visuellen Verarbeitungs- } \\
\text { geschwindigkeit } \\
\text { - Störungen in der Bewe- } \\
\text { gungswahrnehmung } \\
\text { - Sehstörungen }\end{array}$ & $\begin{array}{l}\text { - Halluzinationen } \\
\text { - Aggressivität } \\
\text { - Rücksichtslosigkeit } \\
\text { - Erhöhtes Risikoverhalten } \\
\text { - Vegetative Symptome } \\
\text { (Benommenheit, Schwindel, } \\
\text { orthostatische Hypotonie) } \\
\text { - Tagesmüdigkeit } \\
\text { - Medikamentös induzierte } \\
\text { Psychosen }\end{array}$ \\
\hline
\end{tabular}




\section{Medikation}

Die Fahrkompetenz von Parkinson-Patienten kann durch eine leitliniengerechte pharmakologische Behandlung unterstützt werden. Zu beachten sind jedoch die verschiedenen medikamentösen Nebenwirkungen wie Tagesmüdigkeit, „sudden onset of sleep“, Angst und Depression sowie Impulskontrollstörungen, die kritische Faktoren für die Verkehrssicherheit darstellen [31-33]. Über diese Auswirkungen auf die Fahrtüchtigkeit muss der Patient ausdrücklich informiert werden [8].

\section{Operative Behandlungsansätze}

Die tiefe Hirnstimulation (THS) führt mehrheitlich zu einer Verbesserung motorischer Funktionen [34]. Besonders bei jüngeren Patienten und milderen motorischen Symptomen besteht eine höhere Wahrscheinlichkeit für das Wiederreichen der Fahrtüchtigkeit [35]. Eine mögliche Verschlechterung kognitiver Funktionen in Zusammenhang mit THS wird kontrovers diskutiert [36, 37]. Es ergeben sich keine besonderen Empfehlungen, in Bezug auf die Beurteilung der Fahrkompetenz zwischen Patienten mit THS und medikamentös behandelten Parkinson-Patienten zu unterscheiden [38].

\section{Neuropsychologische Therapie}

Taktische Fehler nach dem Mehrebenenmodell von Michon [39], die verstärkt bei Parkinson-Patienten auftreten, sind ungeschicktere Fahrspurwechsel und größere Probleme bei Abbiegevorgängen [17, 27, 40]. Diese versuchen Patienten häufig während der Fahrt durch ein vorsichtigeres Fahrverhalten auszugleichen [27, 41]. Auf der operationalen Ebene zeigen sich Schwierigkeiten unter anderem durch Probleme beim konsequenten Geschwindigkeitshalten, längerem Zögern bei Wendemanövern oder dem zeitgerechten Bremsen an Ampeln [17, 27, 40, 41]. Eine Vielzahl an Studien weist darauf hin, dass Parkinson-Patienten auf strategischer Ebene Schwierigkeiten haben, ihre Fahrfähigkeiten und ihr -verhalten akkurat einzuschätzen [27, 40, 42]. Einen Überblick zu konkreten Therapieempfehlungen orientiert am Mehrebenenmodell liefern Aschenbrenner und Kollegen [43]. Demnach gilt das Fahrsimulator-Training als eine der bevorzugten therapeutischen Interventionen bei Einschränkungen auf taktischer und operationaler Ebene. Erste Studien zeigen einen potenziellen Nutzen dieser Trainings, Nachweise zu Langzeiteffekten fehlen jedoch $[44,45]$. Um Patienten darüber hinaus über Einschränkungen ihrer Fahrkompetenz zu informieren, bietet die Mobilitätsberatung einen vielversprechenden Ansatz [46].
FAZIT

Parkinson-Patienten sind mit Voranschreiten der Erkrankung durch Kumulationen vielfältiger Beeinträchtigungen erheblich in ihrer Teilhabe am Straßenverkehr eingeschränkt. Jedoch fehlen konkrete Unfallstatistiken, die die Identifikation der Unfallursachen erlauben würden. Nach aktuellem Stand ist vor allem eine Optimierung der medikamentösen Behandlung unter dem Aspekt der Fahrkompetenz entscheidend. Spezifische Trainingsverfahren scheinen hilfreich zu sein, sind aber noch nicht ausreichend evaluiert.

Interessenkonflikt

\section{Erklärung zu finanziellen Interessen}

Forschungsförderung erhalten: nein; Honorar/geldwerten Vorteil für Referententätigkeit erhalten: nein; Bezahlter Berater/interner Schulungsreferent/Gehaltsempfänger: nein; Patent/Geschäftsanteile/Aktien (Autor/Partner, Ehepartner, Kinder) an Firma (Nicht-Sponsor der Veranstaltung): nein; Patent/Geschäftsanteile/Aktien (Autor/Partner, Ehepartner, Kinder) an Firma (Sponsor der Veranstaltung): nein.

Erklärung zu nicht finanziellen Interessen

Die Autoren geben an, dass kein Interessenkonflikt besteht.

Korrespondenzadresse

\author{
Vanessa Andelfinger \\ SRH Klinikum Karlsbad-Langensteinbach \\ Guttmannstr. 1 \\ 76307 Karlsbad-Langensteinbach \\ Deutschland \\ Tel. 07202/617067 \\ vanessa.andelfinger@srh.de
}

Literatur

[1] Buhmann C, Vesper J, Oelsner H. Fahreignung bei Morbus Parkinson. Fortschr Neurol Psychiatr 2018; 86: 43-48. doi:10.1055/s-0043-110051

[2] Buhmann C, Gerloff C. Autofahren bei Morbus Parkinson. Akt Neurol 2013; 40: 315-320. doi:10.1055/s-0033-1349884

[3] Stentzel U, Piegsa J, Fredrich D, et al. Accessibility of general practitioners and selected specialist physicians by car and by public transport in a rural region of Germany. BMC Health Serv Res 2016; 16: 587. doi:10.1186/s12913-016-1839-y

[4] Holmes JD, Alvarez L, Johnson AM, et al. Driving with Parkinson's Disease: Exploring Lived Experience. Parkinsons Dis 2019; 2019. doi:10.1155/2019/3169679

[5] Uc EY, Rizzo M, Johnson AM, et al. Road safety in drivers with Parkinson disease. Neurology 2009; 73: 2112-2119. doi:10.1212/WNL.0b013e3181c67b77

[6] Thompson T, Poulter D, Miles C, et al. Driving impairment and crash risk in Parkinson disease: A systematic review 
and meta-analysis. Neurology 2018; 91: e906-e916. doi:10.1212/WNL.0000000000006132

[7] Brenner-Hartmann J. Begutachtungsleitlinien zur Kraftfahreignung. Kommentar. Schriftenreihe Fahreignung. Bonn: Kirschbaum Verlag; 2018

[8] Peitz J, Hoffmann-Born H. Arzthaftung bei problematischer Fahreignung. Bonn: Kirschbaum Verlag; 2008

[9] Devos H, Vandenberghe W, Tant M, et al. Driving and off-road impairments underlying failure on road testing in Parkinson's disease. Mov Disord 2013; 28: 1949-1956. doi:10.1002/mds.25701

[10] Singh R, Pentland B, Hunter ], et al. Parkinson's disease and driving ability. J Neurol Neurosurg Psychiatry 2007; 78: 363-366. doi:10.1136/jnnp.2006.103440

[11] Pfeiffer RF. Non-motor symptoms in Parkinson's disease. Parkinsonism Relat Disord 2016; 22 Suppl 1: S119-22. doi:10.1016/j.parkreldis.2015.09.004

[12] Ranchet M, Broussolle E, Poisson A, et al. Relationships between cognitive functions and driving behavior in Parkinson's disease. Eur Neurol 2012; 68: 98-107. doi:10.1159/000338264

[13] Rektorova I. Current treatment of behavioral and cognitive symptoms of Parkinson's disease. Parkinsonism Relat Disord 2019; 59: 65-73. doi:10.1016/j.parkreldis.2019.02.042

[14] Uc EY, Rizzo M, O'Shea AM], et al. Longitudinal decline of driving safety in Parkinson disease. Neurology 2017; 89: 1951-1958. doi:10.1212/WNL.0000000000004629

[15] Uc EY, Rizzo M, Anderson SW, et al. Driving under low-contrast visibility conditions in Parkinson disease. Neurology 2009; 73: 1103-1110. doi:10.1212/WNL.0b013e3181bacf6e

[16] Ranchet M, Broussolle E, Paire-Ficout L. Longitudinal Executive Changes in Drivers with Parkinson's Disease: Study Using Neuropsychological and Driving Simulator Tasks. Eur Neurol 2016; 76: 143-150. doi:10.1159/000448992

[17] Amick MM, Grace J, Ott BR. Visual and cognitive predictors of driving safety in Parkinson's disease patients. Arch Clin Neuropsychol 2007; 22: 957-967. doi:10.1016/j. acn.2007.07.004

[18] Ranchet M, Morgan JC, Akinwuntan AE, et al. Visual search and target detection during simulated driving in Parkinson's disease. Accid Anal Prev 2020; 134. doi:10.1016/j. aap.2019.105328

[19] Uc EY, Rizzo M. Driving and neurodegenerative diseases. Curr Neurol Neurosci Rep 2008; 8: 377-383. doi:10.1007| s11910-008-0059-1

[20] Toepper M, Falkenstein M. Driving Fitness in Different Forms of Dementia: An Update. J Am Geriatr Soc 2019; 67: 2186-2192. doi:10.1111/jgs. 16077

[21] Weil RS, Schrag AE, Warren JD, et al. Visual dysfunction in Parkinson's disease. Brain 2016; 139: 2827-2843. doi:10.1093/brain/aww175

[22] Crizzle AM, Classen S, Uc EY. Parkinson disease and driving: an evidence-based review. Neurology 2012; 79: 2067-2074. doi:10.1212/WNL.0b013e3182749e95

[23] Voss H. Zur Beurteilung der Fahrtauglichkeit bei Morbus Parkinson. Nervenheilkunde 2015; 34: 154-160. doi:10.1055/s-0038-1627570

[24] Classen S, Brumback B, Crawford K, et al. Visual Attention Cut Points for Driver Fitness in Parkinson's Disease. OTJR (Thorofare N J) 2019; 39: 257-265. doi:10.1177/1539449219836689
[25] Devos H, Vandenberghe W, Nieuwboer A, et al. Validation of a screening battery to predict driving fitness in people with Parkinson's disease. Mov Disord 2013; 28: 671-674. doi: $10.1002 / \mathrm{mds} .25387$

[26] Barrash J, Stillman A, Anderson SW, et al. Prediction of driving ability with neuropsychological tests: demographic adjustments diminish accuracy. J Int Neuropsychol Soc 2010; 16: 679-686. doi:10.1017/S1355617710000470

[27] Cordell R, Lee HC, Granger A, et al. Driving assessment in Parkinson's disease -a novel predictor of performance? Mov Disord 2008; 23: 1217-1222. doi:10.1002/mds.21762

[28] Uc EY, Rizzo M, Anderson SW, et al. Impaired navigation in drivers with Parkinson's disease. Brain 2007; 130: 24332440. doi:10.1093/brain/awm178

[29] Thiri Kyaw W, Nishikawa N, Moritoyo T, et al. Evaluating the driving ability in patients with Parkinson's disease using a driving simulator. Internal medicine 2013; 52: 871-876. doi:10.2169/internalmedicine.52.9292

[30] Aksan N, Hacker SD, Sager L, et al. Correspondence between Simulator and On-Road Drive Performance: Implications for Assessment of Driving Safety. Geriatrics 2016; 1: 1-9. doi:10.3390/geriatrics1010008

[31] Meindorfner C, Körner Y, Möller JC, et al. Driving in Parkinson's disease: mobility, accidents, and sudden onset of sleep at the wheel. Mov Disord 2005; 20: 832-842. doi:10.1002/ mds.20412

[32] Höglund A, Hagell P, Broman J-E, et al. A 10-Year Follow-Up of Excessive Daytime Sleepiness in Parkinson's Disease. Parkinsons Dis 2019; 2019. doi:10.1155/2019/5708515

[33] Reiff J, Jost WH. Drug-induced impulse control disorders in Parkinson's disease. J Neurol 2011; 258: 323-327. doi:10.1007/s00415-011-5932-1

[34] Rizzone MG, Fasano A, Daniele A, et al. Long-term outcome of subthalamic nucleus DBS in Parkinson's disease: from the advanced phase towards the late stage of the disease? Parkinsonism Relat Disord 2014; 20: 376-381. doi:10.1016/j. parkreldis.2014.01.012

[35] Buhmann C, Vettorazzi E, Oehlwein C, et al. Impact of Deep Brain Stimulation on Daily Routine Driving Practice in Patients with Parkinson's Disease. Parkinsons Dis 2015: 1-9. doi:10.1155/2015/608961

[36] Witt K, Daniels C, Reiff J, et al. Neuropsychological and psychiatric changes after deep brain stimulation for Parkinson's disease: a randomised, multicentre study. The Lancet Neurology 2008; 7: 605-614. doi:10.1016/S14744422(08)70114-5

[37] Aybek S, Gronchi-Perrin A, Berney A, et al. Long-term cognitive profile and incidence of dementia after STN-DBS in Parkinson's disease. Mov Disord 2007; 22: 974-981. doi: $10.1002 / \mathrm{mds} .21478$

[38] Buhmann C, Gerloff C. Could deep brain stimulation help with driving for patients with Parkinson's? Expert Rev Med Devices 2014; 11: 427-429. doi:10.1586/17434440.2014. 929495

[39] Michon JA. Dealing with danger. Summary report of a workshop in the traffic reasearch center 1979

[40] Heikkilä VM, Turkka J, Korpelainen J, et al. Decreased driving ability in people with Parkinson's disease. J Neurol Neurosurg Psychiatry 1998; 64: 325-330. doi:10.1136/jnnp.64.3.325

[41] Stolwyk RJ, Triggs TJ, Charlton JL, et al. Effect of a concurrent task on driving performance in people with Parkinson's disease. Mov Disord 2006; 21: 2096-2100. doi:10.1002/ mds. 21115 
[42] Crizzle AM, Myers AM, Almeida QJ. Self-regulatory practices of drivers with Parkinson's disease: accuracy of patient reports. Parkinsonism Relat Disord 2013; 19: 176-180. doi:10.1016/j.parkreldis.2012.09.003

[43] Aschenbrenner S, Schale A, Weisbrod M, et al. Wiedererlangung der Fahrkompetenz. Behandlungsziel bei psychischen Erkrankungen. Nervenheilkunde 2013; 32: 376-380

[44] Devos H, Morgan JC, Onyeamaechi A, et al. Use of a driving simulator to improve on-road driving performance and cognition in persons with Parkinson's disease: A pilot study. Aust Occup Ther J 2016; 63: 408-414. doi:10.1111/14401630.12263

[45] Teasdale N, Simoneau M, Hudon L, et al. Older Adults with Mild Cognitive Impairments Show Less Driving Errors after a Multiple Sessions Simulator Training Program but Do Not Exhibit Long Term Retention. Front Hum Neurosci 2016; 10. doi:10.3389/fnhum.2016.00653
[46] Surges F. Kognitive Störungen und Verkehrssicherheit. Heft 303. Berichte der Bundesanstalt für Straßenwesen M, Mensch und Sicherheit. Bremen: Fachverlag NW in der Carl Schünemann Verlag GmbH; 2020

Bibliografie

Nervenheilkunde 2021; 40: 359-364

DOI 10.1055/a-1298-1151

ISSN 0722-1541

(C) 2021. Thieme. All rights reserved.

Georg Thieme Verlag KG, Rüdigerstraße 14,

70469 Stuttgart, Germany 


\section{Punkte sammeln auf CM/F.thieme.de}

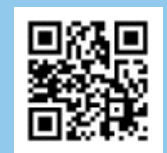

Diese Fortbildungseinheit ist bis zu 12 Monate online für die Teilnahme verfügbar.

Den genauen Einsendeschluss finden Sie beim Modul auf https://cme.thieme.de/CXGZBEN.

Sollten Sie Fragen zur Online-Teilnahme haben, finden Sie unter https://cme.thieme.de/hilfe

eine ausführliche Anleitung. Wir wünschen viel Erfolg beim Beantworten

der Fragen!

Unter https://eref.thieme.de/CXGZBEN oder über den QR-Code kommen Sie

direkt zum Artikel.

VNR 2760512021160213660

\section{Frage 1}

Welche Aussage zu rechtlichen Aspekten der Fahreignung bei Parkinson-Patienten ist richtig?

A Eine umfassende Dokumentation der Aufklärung des Patienten ist nur in Ausnahmefällen erforderlich.

B Bei Parkinson-Patienten ist auch die Eignung für das Führen von Fahrzeugen der Gruppe 2 gegeben.

C Eine extrapyramidale Erkrankung kann eine Herabsetzung der Leistungs- und Belastungsfähigkeit zur Folge haben.

D Bei Zweifeln an der Fahreignung besteht eine Meldepflicht an die Führerscheinbehörde.

E Die Fahrerlaubnis ist ausschließlich von der körperlichen Eignung einer Person abhängig.

\section{Frage 2}

Welche Aussage ist korrekt, wenn Zweifel an der Fahreignung des Patienten bestehen?

A Medizinisch-psychologische Untersuchungen erfolgen ausschließlich bei der Führerscheinbehörde.

B Es ist Aufgabe des Behandlers, den Patienten ausreichend über bestehende Zweifel aufzuklären und zu informieren.

C Bestehen Zweifel an der Fahreignung eines Patienten, muss dieser immer in einjährigen Abständen zur Nachuntersuchung.

D Im rechtlichen Kontext hat das Nichtbefolgen gegebener Empfehlungen keine Auswirkungen auf den Patienten.

E Der Behandler kann für eine unzureichende Aufklärung nie haftbar gemacht werden.

\section{Frage 3}

Welche Aussage zu Parkinsonsymptomen und Fahrkompetenz trifft zu?

A Entscheidungen über die Fahrkompetenz fallen aufgrund eines etablierten und standardisierten Verfahrens.

B Die Hoehn \& Yahr Skala gibt Aufschluss über den Schweregrad der psychischen Erkrankung bei Parkinson-Patienten.

C Das Beachten mehrerer Reize ist für Parkinson-Patienten immer problemlos möglich.

D Neben den motorischen Symptomen sind auch die nicht motorischen Symptome für die Bewertung der Fahrkompetenz relevant.

E Die Beeinträchtigung der Fahrkompetenz bei Demenzerkrankungen ist nicht von deren Schweregrad abhängig.

\section{Frage 4}

Welche Aussage zu den visuellen Symptomen bei Parkinson-Patienten ist korrekt?

A Eine wesentliche visuelle Funktionsstörung ist die Verschlechterung der Kontrastsensitivität.

B Anforderungen an die visuelle Leistungsfähigkeit sind nicht in der Fahrerlaubnisverordnung (FeV) geregelt.

C Insbesondere Verschlechterungen im Bereich der Farbwahrnehmung führen zu einer schlechteren Fahrzeugkontrolle und langsameren Reaktionszeiten.

D Störungen der Bewegungswahrnehmung treten bei Parkinson-Patienten nie auf.

E Bei Parkinson-Patienten bestehen immer auch optische Halluzinationen.

- Weitere Fragen auf der folgenden Seite ... 


\section{Punkte sammeln auf CME.thieme.de}

Fortsetzung $\ldots$

\section{Frage 5}

Welche Aussage zur Prüfung der Fahreignung mit Fahrsimulatoren ist richtig?

A Die Quote des Nichtbestehens bei Parkinson-Patienten ist im Fahrsimulator im Vergleich zur Fahrverhaltensbeobachtung höher.

B Fahrsimulatoren besitzen immer die gleiche Ausstattung.

C Durch Fahrsimulatoren kann ein direktes Unfallrisiko simuliert werden.

D Bei älteren Fahrern sind Fahrsimulatoren kaum als Maße der Fahrleistung geeignet.

E Eine Reproduktion der immer gleichen Fahrstrecke ist im Fahrsimulator nicht möglich.

\section{Frage 6}

Welche Aussage gilt für Patienten und Behandler im Kontext der Fahreignung von Parkinson-Patienten?

A Die Schweigepflicht darf unter keinen Umständen gebrochen werden.

B Aufgabe des Arztes ist es auch, dem Patienten seine Eigenverantwortung aufzuzeigen.

C Bei visuellen Problemen ist die Beurteilung durch einen neurologischen Facharzt ausreichend.

D Ist eine motorische Eignung gegeben, müssen weitere Leistungsbereiche nicht mehr untersucht werden.

E Zur Beurteilung der Fahreignung des Patienten kann sich der Behandler ausschließlich auf seine klinische Erfahrung stützen.

\section{Frage 7}

Welche Aussage zu den kognitiven Symptomen, die Zweifel an der Fahrkompetenz von Parkinson-Patienten begründen können, trifft zu?

A Parkinson-Patienten zeigen häufig Störungen im Bereich verschiedener Aufmerksamkeitsleistungen.

B Störungen in der Verarbeitung räumlich-visueller Informationen sind nicht relevant für die Fahrkompetenz bei Parkinson-Patienten.

C On-/Off-Phänomene gehören in die Reihe der kognitiven Erkrankungen bei Parkinson-Patienten.

D Eine kognitive Verlangsamung tritt bei Parkinson-Patienten nie auf.

E Bereits leichte Demenzen führen immer dazu, dass ParkinsonPatienten nicht mehr fahrtüchtig sind.

\section{Frage 8}

Welche Nebenwirkung einer pharmakologischen Behandlung stellt keinen kritischen Faktor für die Verkehrssicherheit dar?
A Angst und Depression
B „sudden onset of sleep“
C Impulskontrollstörungen
D Appetitverlust
E Tagesmüdigkeit

\section{Frage 9}

Welche Aussage ist in Hinblick auf die tiefe Hirnstimulation (THS) korrekt?

A Besonders bei stark ausgeprägten motorischen Symptomen wird die Aufnahme der Fahrtüchtigkeit in allen Fällen wieder erreicht.

B Eine Verschlechterung kognitiver Symptome bei THS ist völlig ausgeschlossen.

C Im Kontext der Beurteilung der Fahrkompetenz ist die THS deutlich von der pharmakologischen Behandlung zu unterscheiden.

D Die THS garantiert in allen Fällen ein Wiedererlangen der Fahrkompetenz.

E Die THS kann vor allem zu einer Verbesserung motorischer Symptome führen.

\section{Frage 10}

Welche Aussage im Kontext der neuropsychologischen Therapie der Fahreignung bei Parkinson-Patienten trifft zu?

A Ungeschickte Fahrspurwechsel sind nur im Realverkehr trainierbar.

B Parkinson-Patienten sind nie in der Lage, Schwierigkeiten auf taktischer und operationaler Ebene im Fahrprozess auszugleichen.

C Parkinson-Patienten können ihre Fahrfähigkeit und ihr -verhalten immer genau einschätzen.

D Das Training mit dem Fahrsimulator kann Patienten besonders bei Schwierigkeiten auf taktischer und operationaler Ebene helfen.

E Für das Fahrsimulatortraining ist der Langzeitnutzen nachgewiesen. 